\title{
Le rôle des ouvriers et entrepreneurs britanniques dans le décollage industriel français des années 1820
}

The part of British workers and contractors

in the French industrial take-off of the 1820's

Michel Cotte

\section{OpenEdition}

\section{Journals}

Édition électronique

URL : http://journals.openedition.org/dht/1346

DOI : $10.4000 /$ dht 1346

ISSN : 1775-4194

Éditeur :

Centre d'histoire des techniques et de l'environnement du Cnam (CDHTE-Cnam), Société des élèves du CDHTE-Cnam

\section{Édition imprimée}

Date de publication : 1 décembre 2010

Pagination : 119-130

ISBN : 978-2-9530779-5-7

ISSN : 0417-8726

\section{Référence électronique}

Michel Cotte, « Le rôle des ouvriers et entrepreneurs britanniques dans le décollage industriel français des années 1820 », Documents pour l'histoire des techniques [En ligne], 19| $2^{\mathrm{e}}$ semestre 2010, mis en ligne le 21 juin 2011, consulté le 07 septembre 2020. URL : http://journals.openedition.org/dht/1346 DOI : https://doi.org/10.4000/dht.1346 


\title{
Le rôle des ouvriers et entrepreneurs britanniques dans le décollage industriel français des années 1820
}

\author{
Michel Cotte \\ Centre François Viète \\ Université de Nantes
}

a période qui suit la fin du Blocus continental et
le Congrès de Vienne marque une recrudescence rapide des affaires internationales, notamment entre le Continent et les îles Britanniques. Suscité par les premiers voyageurs', l'intérêt pour la technique et les capacités industrielles de la Grande-Bretagne est vif, dès la paix en vigueur. II s'apparente à une véritable découverte de I'avance prise dans ces domaines, pendant que la guerre faisait rage en Europe, par les milieux d'affaires et les entrepreneurs continentaux, tout particulièrement en France. Beaucoup d'entre eux sont avides de découvrir les réalisations d'Outre-Manche, le machinisme, les objets produits mécaniquement, etc., tout autant que de saisir les opportunités d'affaires offertes par la situation. Jacques Payen a été l'un des premiers, à propos des machines à vapeur, à souligner combien le décalage technique s'était creusé entre les deux rives de la Manche durant la Révolution et I'Empire².

Cette opinion anglophile est également partagée par les milieux aristocratiques, dont certains membres éminents avaient été des émigrés en Angleterre, à commencer par Louis XVIII en personne. Elle recouvre la recherche d'affaires nouvelles, d'investissements, mais aussi de modèles de développement économique et elle se traduit par une attitude politique plutôt favorable aux transferts techniques venus d'Outre-Manche, dans les allées du pouvoir. Plusieurs entrepreneurs sauront en

1 Charles Dupin, Voyages dans la Grande-Bretagne entrepris relativement aux services de la Guerre, de la Marine et des Ponts et Chaussées de 1816 à 1819, six volumes avec atlas, Paris 1821-1822, réédition 1826 ; M. Bradley et F. Perrin, "Charles Dupin's Visits to the British Isles, 1816-1824 », Technology and Culture, 1991, 32-1, p. 47-68; C. Christen et F. Vatin (dir.), Charles Dupin (1784-1873)..., Rennes, PUR 2009.

2 Jacques Payen, La machine à vapeur fixe en France, Paris, CTHS, 1985. tirer profit, comme les Seguin avec le duc de Noailles et le baron de Vitrolles ${ }^{3}$.

Les services de l'État, l'Académie, les corps d'ingénieur ne sont pas en reste et ils organisent rapidement un ensemble de voyages d'études que nous avons déjà eu l'occasion de présenter ${ }^{4}$, sur des filières techniques nouvelles ou en cours de renouvellement: les chemins de fer ${ }^{5}$, les ponts suspendus ${ }^{6}$, les bateaux à vapeur américains ${ }^{7}$ par exemple. Le résultat forme une documentation assez complète dans un domaine donné, présentée à l'Académie ou dans les instances du corps commanditaire du voyage. Une version imprimée est généralement éditée assez rapidement, à même de fournir des informations intéressantes et utiles, à condition de disposer sans trop tarder du précieux rapport.

Cette ambiance générale s'accompagne à l'occasion de véritables modes, avant même que la technique en question soit adaptée sur le Continent. À cet égard, la question des ponts suspendus est un bon exemple. Un premier compte rendu paraît en 1815, traduction d'un petit article américain publié en Pennsylvanie à propos d'une réalisation pionnière locale, la passerelle de Schuylkill River ${ }^{8}$. Il passe inaperçu des contemporains

3 Michel Cotte, Le choix de la révolution industrielle, les entreprises de Marc Seguin et ses frères, Rennes, PUR 2007, p. 209-210.

4 Michel Cotte, De l'espionnage industriel à la veille technologique, Belfort, Besançon, Université technologique Belfort Montbéliard, Presses universitaires de Franche-Comté, 2005, chapitre 1

5 G. de Gallois, "Des chemins de fer en Angleterre », Annales des Mines, 1818, p. 37-144.

6 Claude-Louis Navier, Rapport et mémoire sur les ponts suspendus, Paris, 1823, 2éd., 1830.

$7 \mathrm{~J}$.-B. Marestier, Mémoire sur les bateaux à vapeur des ÉtatsUnis d'Amérique, Paris, 1824.

8 Bulletin de la Société d'encouragement pour l'industrie natio- 
français ; c'est encore trop tôt pour ce sujet, pourtant développé depuis de début des années 1800 OutreAtlantique et tout récemment Outre-Manche. II en va tout différemment à compter de 1821-1822 où les journaux ${ }^{9}$ et quelques livres ${ }^{10}$, parfois un peu plus anciens ${ }^{11}$, s'emparent de cette question et la mettent sur le devant de la scène. II faut dire que la situation a bien changée : les Anglais ont entrepris de spectaculaires projets ${ }^{12}$, les Ponts et chaussées ont envoyé l'un de leurs meilleurs ingénieurs, Navier, enquêter sur le terrain des réalisations britanniques ${ }^{13}$, et quelques entrepreneurs ou ingénieurs français envisagent des projets.

\section{Les entreprises privées : de la recherche d'affaires à l'organisation des transferts}

Les historiens de l'économie ont souvent, et parfois brillamment, comparé les économies britannique et française, notamment à la fin de l'Époque moderne, comme le firent François Crouzet $^{14}$ et, d'une manière plus indirecte, Fernand Braudel ${ }^{15}$. II en va de même pour les dynamiques propres de la première industrialisation, où les travaux de Thomas S. Ashton ${ }^{16}$ ou de David S. Landes ${ }^{17}$ ont marqué durablement I'historiographie. C'est toutefois John R. Harris qui a le premier centré l'étude des échanges et des transferts de technologie sur la question de la recherche des savoirs étrangers, notamment par la migration des compétences. Ses articles, regroupés en deux ouvrages de base, restent le point de départ incontournable et une référence

nale, 1816, 15, p. 278.

9 [Jean-Gabriel Moléon], Annales de l'industrie nationale et étrangère, Paris, mars 1821 ; Le Moniteur, 8 décembre 1821, p. 1651-52, cité par Seguin comme source d'inspiration de son prototype.

$10 \mathrm{~J}$. Cordier, Histoire de la navigation intérieure de la France, de I'Angleterre et des États-Unis, Paris, 1819-1821.

11 Joseph-Michel Dutens, Mémoire sur les travaux publics d'Angleterre, Paris, 1819.

12 J.-G. Moléon, op. cit. ; Pictet et de Candolle, Bibliothèque universelle, Genève [automne] 1822, 21.

13 C-L.. Navier, op. cit.

14 François Crouzet, De la supériorité de l'Angleterre sur la France, l'économique et l'imaginaire XVIIe-XX siècles, Paris, Perrin, 1985.

15 Fernand Braudel, Civilisation matérielle, économie et capitalisme, XVe-XVIII' siècle - 3. Le temps du monde, Paris, Armand, Colin, 1979.

16 Thomas S. Ashton, The Industrial Revolution 1760-1830, 1948, une dizaine de rééditions.

17 David S. Landes, The Prometheus Unbound, Technical Change and Industrial Development in Western Europe from 1750 to Present, London, Cambridge university press, 1969, traduction française 1975. essentielle pour ce type d'approche ${ }^{18}$. Nous avons déjà eu l'occasion de commenter ce travail rigoureux et bien documenté ${ }^{19}$, qui aborde la question du transfert comme une succession d'étapes commençant par le mouvement des idées. II est souvent concrétisé par le déplacement des hommes comme des objets, mais, pour pleinement réussir, il nécessite un environnement approprié, un terreau favorable pour son adaptation. En d'autres termes, après la collecte des idées, la réunion des compétences techniques et de l'outillage sur le terrain de I'entreprise constitue le point de passage incontournable dans la concrétisation du transfert technique.

A partir des travaux $d^{\prime}$ Annie Laurant ${ }^{20}$, prenons l'exemple de la région d'industrie sidérurgique ancienne du Nivernais. Entre 1816 et 1820, plusieurs maîtres de forges de la région ou leurs mandataires font le voyage en Grande-Bretagne, dont Georges Dufaud. L'objectif initial est d'importer rapidement des quantités importantes de fer gallois ou anglais, avant que les droits de douane n'augmentent, ce que tous pressentent voire demandent... Lors de son voyage de 1817, Georges Dufaud signe par exemple un contrat d'achat pour 8000 tonnes de barres de fer, livrables dans l'année, avec le sidérurgiste gallois George Crawshay. Toutefois, et rapidement, ces contacts évoluent vers un intérêt technique marqué et le désir d'effectuer une adaptation des méthodes britanniques en France, notamment chez Dufaud alors commanditaire d'un groupe de sidérurgistes de la Loire et du Nivernais.

La seconde étape se situe au printemps de 1818, lorsque le jeune George Crawshay, 23 ans, effectue à son tour un séjour dans le Nivernais, chez leur client, dans la tradition relativement ancienne des séjours à l'étranger pour la formation des futurs négociants. II travaille dans les ateliers des Dufaud à Trézy, où il a le sentiment de reconnaître des technologies bien proches de celles des forges galloises de sa famille! Toutefois, il se plaît en France et son séjour se prolonge, prenant le tour d'une idylle amoureuse. Au printemps 1819, George Crawshay épouse Louise Dufaud, la fille de Georges Dufaud.

Les relations entre les Dufaud et les Crawshay s'étaient jusque-là limitées aux voyages et aux affaires commerciales. La question du transfert de technologies rencontrait par exemple une réticence de la part des Gallois, en particulier à propos d'une éventuelle venue d'ouvriers à Trézy. Après le mariage, les relations entre

18 John R. Harris, Essays in industry and technology in $18^{\text {th }}$ Century, Aldershot, Ashgate,1992; id., Industrial espionnage and technology transfers, Britain and French in the $18^{\text {th }}$ Century, Aldershot, Ashgate Variorum, 1997.

19 M. Cotte, op. cit., 2005, p. 15-20.

20 Annie Laurant, Des fers de Loire à l'acier Martin, Paris, Royer 1995-1997, 2 vol. 
les deux familles prennent une autre tournure. C'est l'époque où Georges Dufaud et Louis Boigues, son commanditaire financier, projettent l'édification du site de Fourchambault, en suivant les meilleurs critères des sidérurgistes britanniques. Les travaux débutent en 1820.

La troisième étape de coopération entre le groupe Boigues - Dufaud et la famille Crawshay commence précisément en 1820, vraisemblablement favorisée par I'intéressement des Gallois dans le projet de Fourchambault. L'appel à la venue d'ouvriers spécialisés gallois est cette fois suivi d'effet : trois viennent dans l'été, assistés de deux interprètes. En 1823, une arrivée de douze techniciens gallois est notée, directement liée au démarrage des productions de la nouvelle usine. Elles se poursuivent jusqu'en 1827, pour atteindre une cinquantaine d'ouvriers sur le site, sur un total de 250. II s'agit principalement de puddlers, mais aussi d'ingénieurs, de forgerons, de fondeurs, de lamineurs, de mécaniciens et de charpentiers. Un pourcentage proche de $20 \%$ d'ouvriers britanniques se maintient pendant quelques années, alors que les effectifs augmentent régulièrement. Après 1830, cette proportion décroît nettement, sans toutefois tarir le flux des arrivées qui se poursuit jusqu'en 1848 pour I'ensemble du groupe Boigues - Dufaud.

Les immigrants de Fourchambault sont généralement de jeunes ouvriers attirés par de hauts salaires. Souvent, ils viennent juste de se marier, une condition sans doute favorable à leur recrutement dans les ateliers des Crawshay au sud du Pays-de-Galles. Ils arrivent avec leur épouse et leur premier enfant naît fréquemment dans le Nivernais. La communauté britannique de Fourchambault culmine à 150 adultes et le même nombre d'enfants en 1830 ; ils ont leur propre cité ouvrière. Les séjours sont de plusieurs années, mais rarement définitifs. II est vraisemblable qu'un accord prévoyait leur retour, car de nombreux «born in France » sont enregistrés comme vivant dans les districts industriels du Pays de Galles, au milieu du XIXe siècle ${ }^{21}$.

Parallèlement à la venue d'ouvriers gallois et anglais dans le Nivernais, Georges Dufaud poursuit l'importation de machines anglaises et il effectue de très nombreux voyages en Angleterre, jusqu'à une mission officielle pour le gouvernement français en 1832 .

D'une manière générale, la main d'œuvre britannique en France est reconnue de qualité, et elle n'a pas souvent d'équivalent local dans le cours des années 1820, notamment dans la sidérurgie et la mécanique. Mais elle est aussi jugée difficile à stabiliser et onéreuse. Dans cette optique, la tâche prioritaire des patrons français, une fois les installations en place, est la transmission du

21 A. Laurant, op. cit., 1995, p. 110-121. savoir-faire par l'éducation d'ouvriers locaux. Dans sa grande majorité, la main d'œuvre britannique ne tient pas à rester trop longuement en France. Les séjours sont de quelques années, parfois moins. Les motivations principales sont l'argent, par un salaire sensiblement supérieur à celui touché en Angleterre, d'environ 30\%, des primes substantielles au moment du départ pour couvrir les frais de voyage et d'installation. Toutefois, de leur côté, les ouvriers britanniques se plaignent qu'assez vite les engagements pris par leurs entrepreneurs français, ou anglais en France, ne sont pas tenus. On leur demande plutôt de former leurs remplaçants locaux que d'effectuer par eux-mêmes les tâches pour lesquelles on les a recrutés. Cela apporte parfois un ensemble de raisons qui motive un retour plus tôt que prévu22.

\section{Une entreprise anglaise en France durant la Restauration}

La société Manby \& Wilson est certainement l'entreprise d'origine britannique la plus importante qui s'installe en France sous la Restauration. Ses ateliers de Charenton sont en effet rapidement capables de traiter de gros volumes d'affaires, simultanément dans plusieurs domaines voisins : la fonderie de grandes pièces, les semiproduits métallurgiques, la construction des machines à vapeur et même la construction de bateaux métalliques. Jacques Payen les présente comme les continuateurs de la grande tradition de Watt en France ${ }^{23}$, et ils peuvent être considérés, avec les établissements de Chaillot à Paris, dirigés à cette époque par le Britannique Humphrey Edwards $^{24}$, les Frérejean en région lyonnaise ${ }^{25}$ et Cockerill à Liège ${ }^{26}$, comme les précurseurs de la grande industrie métallurgique intégrée sur le continent européen. Aaron Manby, entrepreneur et ingénieur précédemment installé à Birmingham, et Daniel Wilson s'associent pour venir créer en France, en 1819, un grand ensemble d'industrie métallurgique et de construction mécanique, dans le faubourg de Charenton à Paris. Le projet a certainement été supporté par la banque Thuret, qui est à peu près au même moment le promoteur d'un autre grand projet

22 Mechanic's Magazine, vol 2, 1824, chronique des débats de la Commission de la Chambre des Communes sur l'abolition des lois restrictives à l'émigration des ouvriers anglais.

23 Jacques Payen, Capital et machine à vapeur au XVIII' siècle, Paris, Mouton 1969, p. 104-5.

24 II a repris, en 1815, la firme des frères Périer, les premiers constructeurs de machines à vapeur en France à la fin de l'Ancien Régime ; voir J. Payen, op. cit., 1985.

25 Pierre Cayez, Métiers jacquard et hauts fourneaux, aux origines de l'industrie lyonnaise, Lyon, PUL, 1978.

26 Robert Halleux, Cockerill, deux siècles de technologie, Liège, du Perron 2002. 


\section{Le rôle des ouvriers et entrepreneurs britanniques dans le décollage industriel français des années 1820}

de transplantation d'industrie anglaise en France : I'usine de coton d'Ourscamp ${ }^{27}$. Un brevet de " construction de vaisseau et de bateau en fer, et machine à vapeur à cylindre oscillant » est obtenu le 28 mai 1821. Les ateliers de Charenton ouvrent en 1822, dotés de cinq machines à vapeur basse pression, dont la construction pour les ateliers industriels devient l'une de leurs spécialités. Les capacités de production sont rapidement d'une quarantaine de machines par $\mathrm{an}^{28}$.

Lorsqu'Aaron Manby quitte l'Angleterre, c'est un ingénieur déjà connu et il garde d'importants liens avec son pays natal. II est, par exemple, I'un des membres fondateurs de l'Institution des ingénieurs civils de Londres. L'autre lien très actif gardé par cette entreprise avec ses origines anglaises, et qui peut la faire qualifier d'entreprise britannique sur le sol français, est le nombre très élevé d'employés d'Outre-Manche dans ses ateliers. Leur progression suit le développement des ateliers, en lien avec le décollage industriel de cette période : 200 sont indiqués pour $1824^{29}, 250$ en $1825^{30}$, et 350 l'année d'après ${ }^{31}$.

Il ne fait pas de doute que ce type d'entreprise arrive au bon moment en France. De nature franchement industrielle, elle bénéficie d'importants moyens financiers, en particulier pour faire venir un nombre élevé d'ouvriers anglais. II s'agit bien d'une entreprise transplantée, qui dispose de fortes capacités de recrutement et d'information en Angleterre. Presque immédiatement, l'accueil d'une instance officieuse comme la Société d'encouragement pour l'industrie nationale est enthousiaste et l'établissement largement reconnu, par une médaille d'or de première classe décernée dès 1825 . Les termes du rapport dans le Bulletin sont révélateurs de l'ambiance de l'époque :

« L'industrie anglaise a pris des ailes ; elle a passé la mer, elle s'acclimate et s'organise dans plusieurs contrées de la France. L'établissement de MM. Manby et Wilson, dont nous allons rendre un compte sommaire, ne le cède en rien, par ses dimensions et la perfection de ses travaux, aux plus beaux établissements de ce genre que possède la Grande-Bretagne. [...] ajoutons que l'établissement occupe 500 ouvriers, dont la moitié sont français ».

« [...Ils] sont venus généreusement nous apporter leur

27 Serge Chassagne, Le coton et ses patrons, Paris, éd. de I'EHESS 1991, p. 421-2.

28 J. Payen, op. cit., 1969, p. 104.

29 Mechanic's Magazine, 1824, 2, p. 76.

30 Bulletin de la Société d'encouragement pour l'industrie nationale, 1825, p. 123-126.

31 Franklin Journal, 1827, 3, p. 200-201. savoir et leur précieuse expérience dans une branche $d^{\prime}$ 'industrie du premier rang, et qui, en se fixant parmi nous, se sont exposés à des jugements très sévères dans leur pays $»^{32}$.

On note que la présence d'une moitié d'ouvriers français dans une telle compagnie est ressentie comme un fait très positif. Bien entendu, la presse anglo-saxonne et les instances britanniques ne manqueront pas de retenir ce même fait, mais en soulignant que la moitié des effectifs sont d'origine anglaise ${ }^{33}$ !

Début 1826, I'entreprise de Manby \& Wilson prend une nouvelle dimension par le rachat de la Compagnie du Creusot, la grande affaire de sidérurgie au coke montée par la famille Wendel et William Wilkinson, dans les années 1780. Elle connut une histoire technique, commerciale et juridique complexe, n'atteignant pas les résultats escomptés. Manby \& Wilson la rachètent dans un souci d'intégration. Malgré ses déboires passés, ce site présente d'excellentes dispositions naturelles, par la proximité de la houille et du canal du Centre ${ }^{34}$.

Les archives Seguin ${ }^{35}$ donnent une idée du positionnement de Manby \& Wilson sur le marché français dans les années 1820, à deux occasions. La première intervient en 1825-1826, lorsque les Seguin mènent une enquête approfondie pour leurs projets de bateaux à vapeur destinés à remonter le Rhône ${ }^{36}$. L'offre de bateaux en fer équipés de machine à vapeur est la plus complète qui leur est faite en France, mais elle souffre de deux inconvénients majeurs. Elle est jugée bien trop chère et elle reste sur le standard de la machine à vapeur de Boulton - Watt à basse pression, alors que les frères Seguin ont compris que cette solution n'avait pas d'avenir pour la navigation fluviale et qu'il fallait, comme les Américains, regarder résolument vers la haute pression. L'affaire ne se fait pas, les Seguin préférant commander un moteur à vapeur haute pression spécifique aux ateliers londoniens de Martineau \& Taylor.

La seconde rencontre entre les Seguin, cette fois constructeurs de la ligne ferroviaire de Saint-Étienne à Lyon, et Manby \& Wilson concerne le marché des rails ${ }^{37}$. \|l s'agit là d'une âpre compétition, pour laquelle les

32 Molard jeune, «Rapport sur les fonderies et établissements d'industrie de MM. Manby et Wilson à Charenton près de Paris », Bulletin de la Société d'encouragement pour l'industrie nationale, avril 1825, p. 123-126.

33 William Turner, Mechanic's Magazine, 1824, 2, p. 76, débat sur l'émigration des artisans.

34 Monique Bonnot, Recherches sur la fonderie du Creusot jusqu'en 1814, Faculté des lettres de Paris, 1960, mémoire ms. 35 Archives départementales de l'Ardèche, 41J.

36 M. Cotte, op. cit., 2007.

37 Ibid. 
acheteurs mettent en concurrence à peu près tout ce qui compte à ce moment-là des deux côtés de la Manche, en voulant le dernier cri : des rails en fer laminé de 5 mètres ! Encore plus que dans le cas des bateaux, les firmes britanniques semblent réunir tous les avantages: leur expérience unique du ferroviaire, des prix plus bas malgré le transport et la douane, la qualité du fer puddlé anglais, un équipement en laminoirs alors jugé comme une exclusivité des forges britanniques, la capacité à répondre à un marché énorme pour l'époque d'une ligne de $58 \mathrm{~km}$ à deux voies, soit plus que le Liverpool - Manchester. Après des discussions difficiles, Manby \& Wilson emportent la commande, en exclusivité, pour leur nouveau site du Creusot. II s'agit d'un marché vital qui leur permet de réaliser un équipement en laminoirs puissants, à partir d'un brevet d'importation détenu en exclusivité en France. Ils atteignent alors un prix comparable à celui des Britanniques; en outre ils acceptent des conditions de livraisons très strictes que l'éloignement maritime ne permet pas à leurs anciens compatriotes! Ce marché montre un pas qualitatif et quantitatif dans l'équipement français pour la grande industrie sidérurgique, réalisant un transfert technologique majeur et réussi, car les livraisons de rails tout comme les tests de qualité sont honorés, à la satisfaction du client. Manby \& Wilson traversent toutefois difficilement la crise des années 1830-1832, pour des raisons que nous connaissons mal. Ils sont déclarés en faillite en 1833 et ils doivent liquider leur entreprise. La partie du Creusot est reprise en 1836 par les frères Schneider ${ }^{38}$.

D'autres entrepreneurs peuvent être cités, venant tenter leur chance en France après une première expérience industrielle dans leur pays natal, à I'exemple de Philip Taylor qui, après avoir travaillé pour les Seguin à Londres, envisage de venir à Lyon monter une affaire "en grand», pour finalement s'installer durablement à Marseille ${ }^{39}$. Le cas de James Jackson, installé dans la région de Saint-Étienne dès le début de la Restauration, est également un exemple emblématique de la venue d'un entrepreneur anglais en France, dans le créneau de l'acier fondu, un véritable goulot d'étranglement du développement de I'industrie mécanique, de l'outillage notamment ${ }^{40}$. Le tropisme du pays natal est très fort car il rentre pour

38 Christian Devillers et Bernard Huet, Le Creusot, naissance et développement d'une ville ouvrière, 1792-1914, Champ Vallon, 1981. 39 Olivier Raveux, Marseille, ville des métaux et de la vapeur au XIXe siècle, Paris, CNRS Ed., 1998; id., " Un technicien britannique en Europe méridionale : Philip Taylor (1786-1870)», Histoire, économie et société, 2000, 19-2, p. 253-66.

40 Jean-François Belhoste, Fonte fer acier, Rhône - Alpes, Lyon, L'inventaire - Images du Patrimoine, 1992. ses derniers jours en Angleterre ; mais ses fils restent définitivement en France, poursuivant les entreprises de leur père.

La venue d'entrepreneurs étrangers n'est cependant pas nouvelle, car elle exista sous l'Ancien Régime où elle fut généralement favorisée par le gouvernement ${ }^{41}$. Ce fut même un cas de figure assez répandu de I'histoire économique européenne, à l'Époque moderne, sous la forme d'une association entre la compétence technique, en la personne de l'entrepreneur, et un capital local, parfois le prince lui-même, souvent un groupe de notables. Ce qui caractérise la période des années 1820, c'est le nombre élevé d'entrepreneurs, leur origine quasi exclusivement anglaise et le fait que le financement puisse provenir des premières banques d'affaires en France, comme le groupe Thuret qui se fait une spécialité de ce type d'entreprises franco-britanniques.

\section{D'autres modèles pour le transfert ? De l'usine clé en main à l'adaptation innovante}

Le modèle de l'usine équipée en matériel britannique et animée par une maîtrise et des ouvriers venus d'OutreManche, à l'image de Manby \& Wilson dans la sidérurgie ou d'Ourscamp pour le coton, connaît une réussite, dans les années 1820, qu'il n'avait pas vraiment obtenue sur le sol français à la fin de l'Ancien Régime, notamment dans ces deux domaines. Les travaux de Jacques Payen pour I'industrie de la vapeur ${ }^{42}$ ou de Serge Chassagne pour le $\operatorname{coton}^{43}$, ou encore ceux de Denis Woronoff ${ }^{44}$ montrent un pourcentage notable d'entrepreneurs britanniques à cette période, mais il est cependant minoritaire ou le devient très rapidement. Les entrepreneurs britanniques s'installent tout particulièrement dans la zone allant de la frontière du Nord de la France à la Normandie ; souvent, après une première expérience, ils choisissent la région parisienne; les ports de Nantes et de Bordeaux ont également leurs faveurs, un peu plus tard Marseille.

La question est maintenant de savoir si un ou d'autres modèles de transferts réussis coexistent à cette époque. Du point de vue des entreprises privées, un exemple que nous avons longuement étudié présente une autre approche des relations au monde technique et industriel britannique : les entreprises des frères Seguin ${ }^{45}$. Rappelons

41 Liliane Hilaire-Pérez, L'invention technique au siècle des Lumières, Paris, Albin Michel, 2000.

42 J. Payen, op. cit., 1985.

43 S. Chassagne, op. cit., 1991.

44 Denis Woronoff, Histoire de l'industrie en France, du XVle siècle à nos jours, Paris, Seuil, 1994.

45 Michel Cotte, Le fonds d'archives Seguin, Privas, ADA, 1997 ; id., Innovation et transfert de technologies, les cas des entreprises 
qu'ils sont à l'origine du pont suspendu léger en France et qu'ils furent parmi les premiers en Europe à tenter la navigation à la vapeur au moyen de la haute pression. Les Seguin construisirent également le chemin de fer de SaintÉtienne à Lyon, auquel nous avons vu Manby \& Wilson collaborer. II s'agit d'une ligne très novatrice qui constitua un pendant du Liverpool-Manchester, pour le moins la première ligne ferroviaire dotée de machines locomotives construites en dehors des îles britanniques. Ils pratiquèrent de manière avisée et sélective les différentes variantes de la collecte d'information: les sources imprimées, le voyage d'étude à l'étranger, les relations d'affaires ${ }^{46}$. Comment se distinguent-ils des exemples précédents, notamment dans leurs rapports aux collaborateurs britanniques ? À vrai dire, la frontière est perméable, mais une stratégie de l'entreprise se dégage assez nettement. La collecte d'information est poursuivie par le choix d'une entreprise partenaire, à laquelle on achète en premier lieu des " machines modèles », comme dans leur période textile de l'immédiat après 1815, comme pour les deux machines locomotives de Stephenson en 1827. Le cas des deux moteurs à vapeur de bateaux commandés à Londres est un peu hybride, car Marc Seguin a établi le cahier des charges et donné l'architecture d'ensemble.

Ce qui interpelle dans l'attitude des frères Seguin, c'est leur rapport aux ouvriers anglais. Ils les utilisent mais avec parcimonie et uniquement quand ils s'estiment incapables de faire autrement, peut-être pour de simples raisons financières. Quand un certain William Lloyd, ouvrier britannique ayant travaillé pour les Frérejean, leur offre ses services pour le fonctionnement et la réparation des machines à vapeur de bateau à leur chantier rhodanien d'Andance, cette offre est rapidement déclinée alors que, de manière patente, la situation technique n'est pas si simple à gérer pour les Seguin. Nous apprenons au passage les exigences financières élevées de tels ouvriers, à la recherche d'entreprises en mal d'implantation de techniques britanniques. II réclame le double d'un employé français au sommet de la qualification ouvrière de l'époque, à condition de le trouver bien entendu. Tout est là, et un marché de l'emploi existe manifestement. Les Seguin font un peu plus tard appel à un autre Anglais de la région, pour deux affaires ponctuelles. Charles Collins est tout d'abord chargé d'une mission que I'on peut qualifier de tentative d'espionnage sur le bateau à vapeur de l'Américain Church, leur rival régional qui navigue alors sur la Saône; mais il ne leur apprend

de Marc Seguin, 1815-1835, Lille, Presses universitaires du Septentrion, 1998, 2 vol. ; id., op. cit., 2007.

46 M. Cotte, op. cit., 2005. rien qu'ils ne sachent déjà. II est ensuite utilisé comme intermédiaire dans un contentieux de forge, mais il ne réapparaît plus par la suite dans les affaires des frères.

En définitive, les deux seuls Britanniques à avoir travaillé pour eux de manière significative, pendant quelques semaines, sont les employés de Martineau \& Taylor, envoyés pour le montage de la première machine à vapeur de bateau. Ils repartent rapidement, et lorsque Stephenson leur propose un service équivalent, moins de deux ans après, pour le montage de ses machines locomotives, les entrepreneurs ardéchois refusent, sans la moindre hésitation. L'entreprise a une stratégie d'acquisition du savoir-faire par ses cadres internes et de recours à des artisans extérieurs spécialisés, manifestement de bon niveau en région lyonnaise. Elle se dote rapidement de compétences nouvelles dans de nombreux domaines. Le transfert devient ici une " adaptation innovante » d'idées étrangères, en se basant sur les forces et sur les dynamiques du système sociotechnique régional. L'objet acheté est approprié par son étude, monté, démonté, essayé, mais aussi modifié et adapté au contexte local tant de sa production que de son usage. Des innovations importantes peuvent apparaître, du moins dans les meilleurs cas. Le pont suspendu se trouve doté d'une suspension en câbles de fil de fer plutôt qu'en chaînes forgées; la locomotive Stephenson se voit équipée d'une chaudière tubulaire dès son arrivée dans les ateliers de Perrache, avant que la firme de Newcastle ne s'en inspire à son tour et en retire un succès technique magistral.

S'agit-il d'un exemple exceptionnel, lié à des entrepreneurs hors normes et à une situation régionale très favorable? Sans doute, mais pour une part seulement. Le but d'un George Dufaud et de bien d'autres entrepreneurs est d'arriver à se passer de la main-d'œuvre britannique. Par ailleurs, si la présence de celle-ci est effective en région rhodanienne et stéphanoise, durant la Restauration, dans des secteurs cruciaux, elle est peu nombreuse et elle rencontre rapidement une alternative locale de qualité. Par exemple, l'ingénieur des Mines et entrepreneur Louis Beaunier crée rapidement une aciérie concurrente à celle des Jackson à la Bérardière, et il obtient, dès 1819, la médaille d'or de l'exposition nationale organisée par la Société d'encouragement pour l'industrie, pour sa production d'acier fondu ${ }^{47}$.

47 J.-L. Gras, Histoire économique de la métallurgie de la Loire, Saint-Étienne, 1908 ; Bernard Zellmeyer, « Beaunier sidérurgiste et pionnier du rail », Bulletin du Centre d'Histoire régionale de I'Université de Saint-Étienne, 1978-1, p. 43-54. 
Également en plein décollage industriel, la ville de Mulhouse fournit un autre exemple régional majeur, bien étudié par Florence $\mathrm{Ott}^{48}$. On sait le rôle important qu'elle joua rapidement dans l'industrie cotonnière, la mécanique et les machines à vapeur. La première étape du transfert technique y est très présente, notamment la recherche d'information, mais aussi son évaluation en vue d'en tenter l'expérimentation puis la mise en œuvre locale. Le mouvement collectif des entrepreneurs locaux aboutit à la création de la Société industrielle de Mulhouse, dans les années 1824-1825; à l'image de son modèle parisien, la Société d'encouragement pour l'industrie nationale elle se structure en commissions, elle organise rapidement un réseau international grâce à ses nombreux expatriés, elle favorise les comptes-rendus de voyages d'études par ses membres, elle crée une bibliothèque à la forte présence d'ouvrages allemands et anglais, elle tient des réunions, enfin elle publie un Bulletin ${ }^{49}$ et elle met sur pied des expositions interrégionales avec les industriels bâlois et du Wurtemberg. Bref, elle met en place, d'une manière rapide, une veille technologique efficace dans les domaines qui l'intéressent, tout en en retirant un indice de notoriété important. Là encore, et plus qu'en région stéphanoise et lyonnaise, il n'y a pas de nom britannique au sein de ces milieux professionnels qui semblent parfaitement se satisfaire d'un premier stade du transfert technique, celui des idées et sans doute de "machines pour modèle ", achetées fort chères car leur prix contient ce que l'on appellera plus tard un droit de licence. Rappelons qu'il s'agit d'affaires commerciales en bonnes et dues formes, conclues de plein gré entre les partenaires. Là encore, et à l'échelle d'un bassin industriel, la mise en place et l'adaptation de ces technologies nouvelles se fait par des personnels locaux.

Pour comprendre comment la greffe technique peut avoir lieu, il faut prendre en compte les cultures professionnelles locales ou régionales, parfois fort anciennes. Dans un premier temps, elles prédisposent à la prise en compte d'exemples étrangers; puis elles favorisent les démarches innovantes comme dans la papeterie à Annonay, dans l'artisanat du cuivre lyonnais, dans le Jura horloger ou à Mulhouse ${ }^{50}$. II s'agit parfois de techniciens qui ont voyagé et séjourné à l'étranger, à I'image d'un François Bourdon qui, après une expérience technique et industrielle un peu laborieuse dans la Bourgogne du début des années 1820, est allé travailler plusieurs années dans les chantiers navals et les ateliers

48 Florence Ott, La Société industrielle de Mulhouse (18261876), Strasbourg, Presses universitaires de Strasbourg, 1999.

49 Bulletin de la Société industrielle de Mulhouse, à partir de 1826. 50 M. Cotte, op. cit., 2005, chapitre 4. américains. Puis il séjourne à Liverpool pour revenir en France avec une expérience réellement internationale. C'est à lui que les Schneider confieront, en 1836, la responsabilité technique des usines du Creusot ${ }^{51}$.

\section{Vers des intermédiaires spécialisés dans la veille et le transfert technologique}

L'une des caractéristiques de la période est l'apparition d'intermédiaires patentés dans le transfert technologique, pour favoriser les échanges entre partenaires britanniques et français. Ce n'est pas très nouveau en fait, car les intermédiaires commerciaux sont fort nombreux en Europe tout au long de l'Époque moderne. C'est la base même du négoce en gros, et il y a là une continuité à souligner. Ce qui change, c'est d'une part l'évolution et la spécialisation de plus en plus technique des sujets traités, et c'est d'autre part un service qui est payé et non plus un objet acheté. L'intermédiaire fournit des informations, noue des relations, favorise l'éclosion de projets francobritanniques puis, dans un second stade, il peut aller jusqu'à faire des études et des plans pour ses clients, et il peut même suivre en conseiller la bonne conduite des implantations nouvelles.

Un premier exemple emblématique de ces intermédiaires du transfert technique en provenance du négoce est le courtier Alexandre Andelle. Au début de la Restauration, il conduit des affaires de négoce textile entre la France et la Grande-Bretagne, comme Georges Dufaud pour le commerce des fers. II prend lui aussi la pleine mesure de la montée des éléments technologiques comme facteur d'évolution des marchés. Au cours de plusieurs séjours en Angleterre, dont un assez long en 1818-1819, il étudie la filature et le tissage, au départ une enquête commanditée par le Ministère en charge du commerce.

À son retour, il préconise la création d'entreprises britanniques complètes en France, techniquement dirigées et animées par du personnel britannique, comme cela avait été tenté, mais sans véritable succès, à la fin de I'Ancien Régime. Au début des années 1820, il est I'intermédiaire de deux installations d'usines de coton « clés en main ». La première est une filature mécanisée réalisée avec l'entrepreneur William Eaton et la banque Thuret, à Ourscamp, dans la vallée de l'Oise. La seconde concerne un projet de fabrique de tulles et de broderies mécaniques avec John Heathcoat, dont la réalisation effective à Saint-Quentin s'oriente vers la filature en fin du coton, jugée plus rémunératrice ${ }^{52}$. Le déroulement est très semblable : il organise la visite de l'entrepreneur

51 Olivier Raveux, "François Bourdon, ingénieur autodidacte », La revue, Musée des arts et métiers, 22, 1998, p. 14-21.

52 S. Chassagne, op. cit., 1991. 
anglais en France et la rencontre avec les investisseurs potentiels; il recherche un site industriel approprié, propose le dépôt d'un brevet d'importation, demande d'entrée en franchise des machines, recherche l'appui officieux des services ministériels qu'il connaît bien, etc.

À la suite de ces deux expériences de création d'entreprises " à l'anglaise », Andelle structure son activité d'intermédiaire en nouvelles technologies. II crée une Agence générale pour les manufactures consacrée au commerce des machines textiles, des pièces détachées, des machines à vapeur, etc. C'est à lui que les frères Seguin s'adressent lorsqu'ils désirent faire un point précis de l'état des constructeurs de machines à vapeur de bateau en Grande-Bretagne, en 1826. Andelle leur adresse un rapport assez complet du marché et des tarifs pratiqués à Londres, à Liverpool et en Ecosse, ce qui montre l'efficacité de son réseau d'information en Angleterre même ${ }^{53}$. Un peu plus tard, il s'intéresse aux chemins de fer et à leurs implications économiques ${ }^{54}$.

Un second exemple d'intermédiaire technologique nous est fourni par l'ingénieur Marc Isambard Brunel, dans la seconde partie de sa carrière, alors qu'il est installé à Londres. Immigré d'origine française, il a conquis sa réputation dans le monde des ingénieurs civils et des entrepreneurs anglo-saxons durant le premier quart du XIX siècle, à la suite d'une carrière aventureuse aux ÉtatsUnis, puis dans des tentatives d'industrialisation au profit des équipements militaires britanniques durant les guerres napoléoniennes. II est surtout connu comme étant le père d'Isambard Kingdom Brunel, le fameux ingénieur victorien ${ }^{55}$. Dans les années 1820, il se préoccupe de génie civil et de transport, entreprenant notamment le tunnel sous la Tamise, à Londres, un pari technique risqué que terminera son fils.

Sa position est alors privilégiée pour être un intermédiaire d'affaires particulièrement actif entre la France et l'Angleterre, comme nous l'apprennent ses agendas personnels ${ }^{56}$. II est parfaitement bilingue et c'est un ingénieur autodidacte d'un bon niveau, rompu à toutes sortes de sujets techniques. II connaît fort bien le monde des entrepreneurs britanniques, avec lequel il travaille depuis près de vingt ans et où il entretient de nombreuses relations. Londres est idéalement placée comme arrivée naturelle de tout voyageur continental un peu curieux et intéressé aux affaires. C'est l'une des

53 M. Cotte, op. cit., 2007.

54 Alexandre Andelle, Considérations sur l'essor à donner en France au chemin de fer, Paris, 1833.

55 L.T.C. Rolt, Isambard Kingdom Brunel, London, Penguin books, 1957.

56 Archives de I' Institution of the Civil Engineers, Londres, fonds Brunel, journaux annuels, ms., 1824-1830. bonnes adresses londoniennes à laquelle on ne manque pas de se rendre, parfois pour un simple conseil, un contact ou une recommandation. Son frère, resté en Normandie, I'aide dans ses affaires françaises et il dispose d'un agent personnel à Paris, d'origine anglaise, M. Lees, avec lequel il échange une correspondance régulière. L'industriel et banquier parisien Delessert tient ses comptes en France, où il se rend personnellement à plusieurs reprises dans les années 1820.

Outre ses nombreux clients continentaux ${ }^{57}$, il traite directement avec le gouvernement français de la Restauration, lui vendant deux petits ponts suspendus démontables pour l'lle Bourbon, et le conseillant en lui fournissant des études d'implantation industrielle et de travaux publics. En 1829, il organise à Londres la visite des ateliers de son ami Maudslay pour le duc d'Orléans, le futur roi Louis-Philippe. Ses journaux témoignent d'une intense activité de réseau; il devient l'intermédiaire de nombreuses négociations pour l'achat de matériels techniques et de machines. II intervient également pour les brevets que souhaitent déposer des Continentaux en Angleterre. En retour, il propose ses services auprès des firmes britanniques intéressées par la recherche d'affaires en France et en Europe. Brunel apparaît bien comme un précurseur des futurs «ingénieurs conseils» et autres « cabinets d'ingénieurs».

Ce type de professionnalisation de l'information technique et industrielle devient, peu après, une activité de groupe affichée comme telle, avec par exemple les frères Flachat, en France, dans le courant des années 1830. Ils s'appuient sur de jeunes ingénieurs fraichement sortis de la nouvelle École centrale des arts et manufactures pour réaliser des études complètes, à partir des exemples britanniques les plus réussis, d'abord dans le domaine de la sidérurgie puis dans celui des chemins de fer ${ }^{58}$.

Un troisième exemple d'intermédiaire, l'ingénieur William Steward à Bordeaux, illustre un autre type d'intervention, en lien direct avec l'immigration de techniciens britanniques sur le Continent et au contact des réalités régionales. Sa trajectoire d'ingénieur anglais en France peut se reconstituer assez convenablement ${ }^{59}$. Elle paraît exemplaire d'une belle carrière professionnelle à l'étranger, dans la première moitié du XIXe siècle. Originaire de Liverpool, ce jeune mécanicien se fait remarquer en travaillant à deux des premières machines locomotives anglaises, dans les années 1814-1816, puis sur les ponts métalliques que Telford construit dans sa région

57 M. Cotte, op. cit., 2005, p. 143-146.

58 Alain Auclair, Les ingénieurs de l'équipement en France, Eugène Flachat, Le Creusot, Écomusée de la CUCM, 1999.

59 Archives de I'Institution des ingénieurs civils britanniques, Londres, fiche Steward William, ms. 
natale. En 1818, il est employé par Fawcett, le principal constructeur de bateaux à vapeur de Liverpool. Il est signalé l'année suivante à Bordeaux, pour la première fois, où il participe à la construction du bateau à vapeur « la Gironde », vraisemblablement celui de Church. II devient ensuite " l'inspecteur des machines » de la compagnie de Liverpool à Bordeaux ; en clair il est chargé d'entretenir la machine livrée et de prospecter le marché régional. En 1821, il travaille à Paris, dans les ateliers de Chaillot, où il répare une machine à vapeur de type Edwards, peut-être pour l'un de ses commanditaires bordelais. En 1824, il est à nouveau à Bordeaux, installé sous le titre "d'ingénieur de marine » et toujours agent de Fawcett. II s'occupe de la construction d'une machine à vapeur de 12 chevaux destinée à un bateau commandé par un homme d'affaires de la ville.

En 1825, la carrière française de William Steward prend une nouvelle dimension. II se présente cette fois comme "ingénieur mécanicien à Bordeaux », agent d'affaires et ingénieur d'études capable de suivre la réalisation de toute commande technique pour un client. Il est alors membre actif de la Société d'encouragement pour l'industrie nationale française. A partir de 1827 et durant les années 1830, c'est l'une des chevilles ouvrières des expositions industrielles bordelaises, auxquelles il contribue à donner un caractère franco-britannique. ॥ participe alors à divers projets, dont le fameux pont à chaînes métalliques de Cubzac, sur la Dordogne. Steward garde pendant toute cette longue période française des liens importants avec l'Angleterre, continuant à jouer son rôle d'intermédiaire d'affaires et d'animateur de réseau $d^{\prime}$ information. Se retirant vers 1845 , il rentre toutefois et définitivement à Liverpool.

\section{En Angleterre : des opinions opposées à propos du transfert technique vers le Continent}

Dans le cadre des questions de transferts techniques, en Angleterre même, il faut mentionner la création de I'Institution des ingénieurs civils de Londres qui, au début des années 1820, se structure sous la présidence de Thomas Telford ${ }^{60}$. C'est la première association se référant explicitement à un milieu professionnel des ingénieurs, née dans un pays où, il est vrai, les écoles d'ingénieurs et les corps civils d'État n'existent pas. À l'image de Telford, devenu célèbre dans les travaux publics et la construction des ouvrages d'art métalliques, les membres de l'Institution sont des hommes formés par la pratique et connus pour leur réussite professionnelle au sein d'entreprises privées.

60 Garth Watson, The Civils - The story of the Institution of Civil Engineers, London, ICE 1988.
L'Institution apparaît tout de suite comme un milieu très ouvert sur les affaires internationales, notamment dans les travaux publics et les machines; et nous y retrouvons bon nombre des protagonistes du transfert technique rencontrés jusque-là: Marc Brunel, John Martineau, Aaron Manby sont parmi les fondateurs; Daniel Wilson et William Steward viendront un peu plus tard; et il y a immédiatement des membres étrangers: Suédois, Néerlandais et Français, en la personne du baron Dupin, un peu après des entrepreneurs britanniques en Russie, etc.

L'Institution fonctionne sur le mode des sociétés savantes, avec réunions, bibliothèque, commissions, etc. Tout concourt à démontrer qu'elle forme un maillon important de la circulation de l'information technique, dans les deux sens, et un véritable milieu d'affaires internationales tourné vers les marchés du Continent. L'Institution fait connaître les réalisations des ingénieurs britanniques les plus en vue et propose l'exportation de leur savoir-faire, à l'image de Telford lui-même dont la dernière grande affaire de conseil est à propos du canal Gotha, en Suède et à ce moment-là. En retour, ses membres étrangers, souvent des entrepreneurs ou des ingénieurs britanniques émigrés, lui fournissent rapidement des informations précieuses sur les besoins matériels étrangers, les projets envisagés, les forces en présence, les capacités financières à supporter les projets, etc.

En Angleterre même, I'Institution apparaît comme un lobby très actif de la promotion des idées libérales en matière économique, qui s'oppose aux entrepreneurs désireux de conserver le monopole de leurs productions mécanisées et de leurs marchés d'exportation. Ces derniers s'appuient sur un ensemble législatif issu de la période d'affrontement avec la France révolutionnaire, les «Combination Laws» (1799-1800), qui édictent une réglementation très sévère à l'encontre des ouvriers anglais émigrants, visant à conserver le monopole du savoir-faire sur le sol britannique.

Le débat est devenu très vif entre les entrepreneurs, car les départs de techniciens et d'ouvriers qualifiés se sont multipliés au début des années 1820, notamment vers la France. II devient public lorsque la Chambre des Communes propose de réviser les Combination Laws, en 1824, et les prises de position des uns et des autres sont alors répercutées par la presse britannique ${ }^{61}$. À cette époque, la loi s'applique surtout dans certaines branches jugées

61 Par exemple, I'hebdomadaire Mechanic's Magazine, 1824, $2^{\mathrm{e}}$ semestre ; l'immigration ne constitue qu'une facette de cet ensemble législatif qui concerne le statut de l'ouvrier britannique en général, mais elle fait l'objet d'un débat propre. 


\section{Le rôle des ouvriers et entrepreneurs britanniques dans le décollage industriel français des années 1820}

stratégiques pour l'économie britannique : la sidérurgie, la mécanique, les machines textiles notamment ; l'ouvrier s'expose à de graves sanctions pénales, à son retour, si son ancien patron a porté plainte pour départ illégal. La loi concerne également la prohibition de l'exportation de l'outillage et des machines dans toute une série de métiers jugés sensibles: I'horlogerie, les outils de coupe et d'estampage, les tours à fileter, etc. Une commission parlementaire est nommée en 1823-24, pour entendre les différents points de vue des patrons et des ouvriers sur les conséquences de l'émigration pour l'économie anglaise.

Le patronat de Birmingham, spécialisé dans la quincaillerie, l'outillage et les petits objets décoratifs, est particulièrement touché. II se mobilise pour le maintien d'un statut répressif de l'émigration des ouvriers qualifiés. L'un d'eux, Galloway, explique par exemple comment les ouvriers s'éclipsent en douce, sous le prétexte d'une courte absence, pour partir en France. En 1818, il en a par exemple perdu six à huit en quelques jours, profitant de sa propre absence de l'usine. Suite à un meeting public organisé à Birmingham sur la question de I'abrogation, Thomas Osler, de la Chambre de commerce, est envoyé défendre à Londres les intérêts industriels et sociaux de sa cité. II intervient très longuement devant la commission des Communes. Son propos consiste à démontrer que le départ de seulement quelques hommes d'une entreprise, jusque-là saine, peut la compromettre définitivement, car ils emportent un secret de fabrication, parfois un simple tour de main décisif ou bien les plans d'une machine spécialisée. En fait, ce sont les capacités d'exportation en produits finis des entreprises de Birmingham vers l'Europe qui sont compromises par le transfert de certains savoir-faire, notamment vers la France. Ces hommes félons y trouvent un environnement légal et des approvisionnements plus favorables qu'en Angleterre, l'absence de droits de douane. II cite le cas des manufactures de boutons de Birmingham qui, à I'en croire, sont en passe d'être éliminées du marché européen par les nouvelles entreprises parisiennes, aidées de techniciens anglais. Dix-neuf à vingt usines de sa ville sont menacées, et il rappelle que les manufactures de petits objets d'ennoblissement textile ou de décoration font vivre de 130000 à 150000 personnes en Angleterre. Osler stigmatise tout particulièrement le rôle de différents intermédiaires étrangers spécialisés dans le débauchage.

L'Institution des ingénieurs civils est le principal défenseur de l'abrogation des lois contre l'émigration. Son porte-parole devant la commission est l'entrepreneur londonien John Martineau, déjà rencontré. II tente d'obtenir une ouverture libérale des frontières aux ouvriers et aux techniciens britanniques qui sont étroitement associés aux marchés gagnés à l'étranger, de créations d'usines, d'exportations de machines et de chantiers de travaux publics. Plusieurs firmes anglaises importantes doivent beaucoup à l'exportation de machines, à commencer par Boulton \& Watt dans les machines à vapeur, devenue une firme mondialement connue. Beaucoup d'entreprises comme la sienne attendent un développement de ces marchés étrangers, en France tout particulièrement où il a déjà vendu des machines à gaz et des machines à vapeur à cylindre horizontal. C'est le cas d'autres ateliers de moteurs à vapeur et de mécanique comme ceux des Stephenson à Newcastle, de Maudslay à Londres, de Fawcett à Liverpool, de Donkin pour les machines à papier et de nombreux fabricants de machines textiles. L'avenir est dans la recherche de tels marchés à hauts profits, sur le Continent, et les lois protectionnistes en vigueur les pénalisent en empêchant les ouvriers britanniques d'aller correctement installer ces machines.

Par son appartenance aux différents milieux industriels londoniens, John Martineau est représentatif $d^{\prime}$ 'une tendance pragmatique en affaires, favorable à une ouverture des frontières pour les hommes comme pour les marchandises. Outre son statut de membre fondateur des « Civil Engineers », il est aussi membre du « London Mechanics' Institute». Martineau apparaît également comme un homme de réseau. En relations d'affaires avec Brunel, il a aussi des liens avec les industriels britanniques de Paris: Manby, Edwards et Steel. Son affaire marche alors fort bien: 18 machines à vapeur sont en construction dans ses ateliers durant l'été 1825. II apparaît comme un industriel en pleine ascension et un exportateur de machines de premier plan ${ }^{62}$. Pour la commission parlementaire, c'est un homme qui connaît de première main les différentes facettes de la question du départ des techniciens qualifiés vers l'Europe. Les circuits de débauchage depuis Londres vers la France sont généralement animés par des ouvriers anglais expatriés au profit des firmes françaises. Lui même voit pas mal de ses bons ouvriers partir pour les ateliers parisiens, et il en subit un préjudice important. II pense néanmoins que la loi de prohibition agit de manière perverse, allant à l'encontre de ses objectifs. L'ambiance ainsi créée a fait monter les enchères: des pots de vin importants sont versés aux ouvriers débauchés, des salaires anormalement élevés leur sont offerts par les patrons étrangers. Du coup, ce sont les meilleurs qui sont sollicités et qui partent. Une fois sur place, un second effet pervers intervient : beaucoup de ces ouvriers voudraient rentrer

62 Archives de I'Institution des ingénieurs civils, Londres, John Martineau, fiche ms. 
au pays après un ou deux ans en France, mais la loi les pénalise lourdement au retour par des amendes, par l'impossibilité d'obtenir à nouveau un passeport, et cela sur simple dénonciation de leur ancien employeur britannique. La loi invite véritablement à une émigration définitive, alors qu'en fait l'ouvrier anglais n'a pas envie d'abandonner son pays. Le départ est souvent lié à des raisons personnelles momentanées, à un besoin d'argent. Il demande donc de supprimer la loi de prohibition, ce qui aurait deux conséquences majeures. Tout d'abord, les retours ne posant plus de problème, on créera un va et vient permanent d'ouvriers qui ne resteront à l'étranger que pour une durée limitée. Les ouvriers seront satisfaits d'une telle mesure ; ils gagneront un peu d'argent et ils se sentiront plus libres. D'autre part, le marché du travail sera assaini ; et au lieu de voir partir durablement les meilleurs ouvriers, ce sont les moins bons qui auront tendance à s'expatrier et à émigrer définitivement.

Toujours devant la commission parlementaire, Martineau émet également des avis pertinents sur l'efficacité des entreprises françaises et plus largement continentales. II pense que les Britanniques ne sont pas prêts de perdre l'avantage technique qu'ils ont acquis sur le reste du monde, une raison pour lui de ne pas craindre de libéraliser le marché d'exportation des machines. Les Français ont cherché, il est vrai avec un certain succès, à débaucher des ouvriers, à créer des firmes quasiment britanniques sur leur sol capables de reproduire des machines anglaises. Mais il ne faut pas s'en inquiéter outre mesure, car elles sont nettement plus chères tout en restant de qualité inférieure. Cela pour plusieurs raisons : les bons ouvriers britanniques coûteront toujours au moins 25 à $30 \%$ plus cher qu'en Angleterre même ; les matières premières sont beaucoup plus chères en France, jusqu'à $100 \%$ pour la fonte; enfin les transports intérieurs sont médiocres et eux aussi beaucoup plus chers qu'en Angleterre. A I'inverse, un marché libre des machines conduirait à en vendre dans le monde entier ${ }^{63}$. Les libéraux auront gain de cause, les Combination Laws seront abrogées pour le séjour des ouvriers britanniques à l'étranger, rendant possible et légale leur expatriation temporaire.

\section{Conclusion}

Les exemples proposés dans cette étude suggèrent des typologies possibles dans le transfert technique par les entreprises, des caractéristiques régionales, l'importance des questions culturelles ${ }^{64}$. II nous manque toutefois une vision d'ensemble plus large, plus complète et surtout quantifiée. L'impact direct des techniciens britanniques

63 Mechanic's Magazine, vol. cité.

64 M. Cotte, op. cit., 2005. sur le décollage industriel de la France de la Restauration peut être affirmé, ses limites également, mais sa mesure plus précise reste à faire. Plusieurs approches peuvent être suggérées pour cela, par exemple le dépouillement de la presse, l'étude systématique des brevets, l'étude statistique des entrées et sorties de personnes et de machines. Nous avons étudié la question de l'information étrangère dans la pesse professionnelle, sous l'angle statistique et thématique ${ }^{65}$. Dès la fin de l'aventure napoléonienne en Europe, un véritable boom des articles de ce type est manifeste ${ }^{66}$. Toutefois, la collecte d'information est une étape préalable appartenant au domaine de la circulation des idées, et dont l'influence sur la décision de l'entreprise à réaliser un transfert technique, avec ou sans participation directe de machines et de techniciens britanniques reste assez difficile à cerner, même s'il existe des exemples probants.

Les structures nationales encadrant la circulation des idées techniques sont également à prendre en compte, par exemple la politique dissemblable des brevets en France et en Angleterre. À partir des années 1820, les Français font directement appel aux idées techniques étrangères, en proposant une catégorie spécifique de protection: le brevet d'importation ${ }^{67}$. Elle connaît une réussite immédiate. L'effet recherché est clairement de faire venir des entrepreneurs étrangers en leur garantissant une protection légale et la possibilité de développer leurs projets en leur nom propre. Les Britanniques, à la même époque, tendent au contraire à rendre anonymes les idées venues de l'extérieur, car un étranger ne peut pas déposer directement de brevet et il doit passer par un intermédiaire résident permanent, seul nommé sur la patente. Nous avons vu Marc Isambard Brunel proposer ses services dans ce domaine aux Continentaux. Cette politique d'acculturation peut être interprétée de diverses manières. Elle a en tous cas un effet dissuasif, par une protection indirecte, des coûts élevés et une non reconnaissance de l'inventeur étranger. L'effet obtenu est un faible niveau de protection des idées extérieures en Grande-Bretagne, ce qui les rend relativement faciles à adapter. Il est à ce niveau difficile d'estimer le rôle précis joué par les idées étrangères dans le monde britannique, mais il est réel dans un certain nombre de cas comme celui de l'adoption du pont suspendu venu des États-Unis.

Sur le plan du contexte économique général, certaines entreprises anglaises ont du mal à exporter vers le Continent dans de bonnes conditions, alors que les

65 M. Cotte, op. cit., 2005, chapitre 3.

66 Par exemple, le Bulletin de la Société d'encouragement pour I'industrie nationale, années 1814-1815.

67 M. Cotte, op. cit., 2005, chapitre 1. 
affaires intérieures stagnent dans diverses branches. C'est un facteur qui a manifestement pesé en faveur de l'abrogation des lois de prohibition de l'émigration et de l'exportation des machines. C'est une ambiance qui contraste avec l'atmosphère quasiment euphorique du développement de l'industrie en France, dans les années 1820, en partie dû à son protectionnisme sélectif et à son accueil libéral des étrangers. Enfin, la concurrence des productions continentales devient une réalité dans un certain nombre de secteurs: différents domaines de l'industrie textile, les accessoires d'ennoblissement de l'habillement, les ornements et les objets décoratifs notamment, certains secteurs de la quincaillerie, etc.

Nous avons vu que la France exerçait une forte attirance sur beaucoup d'ouvriers et d'ingénieurs britanniques. Elle est vue comme un système économique alternatif, institutionnellement plus attentif à protéger ses cadres techniques de talent car elle en manque. Elle est jugée comme ayant un fort potentiel de croissance, supérieur même à celui de l'Angleterre, et comme une clé d'accès au marché continental dans son ensemble. II s'agit aussi du pays de la seconde chance pour des entrepreneurs qui paraissent condamnés à végéter après un échec ou une demi réussite dans leur patrie d'origine. Ils sont nombreux à voir la France comme un eldorado, d'autant que les capitalistes français, au moins durant les années 1820, n’hésitent pas trop à les rémunérer correctement. Les ouvriers et les techniciens de rang plus modeste voient la France comme un pays qui paye bien et qui permet en quelques années de se constituer un pécule avant de revenir au pays. Une "franco-mania » joue donc un rôle favorable à l'émigration des années 1820, rencontrant celle symétrique de bon nombre d'investisseurs et d'entrepreneurs en France. II faudra la crise économique du début des années 1830, et plus encore celle de 1847-1848, pour marquer un coup d'arrêt à cette immigration importante de techniciens et d'entrepreneurs britanniques en France. 